\title{
Analytical solution of magnetohydrodynamics generalized Burger's fluid embedded with porosity
}

\author{
Kashif Ali Abro ${ }^{1}$ *, Mukarrum Hussain ${ }^{2}$, Mirza Mahmood Baig ${ }^{1}$ \\ ${ }^{1}$ Department of Mathematics, NED University of Engineering Technology, 75270, Karachi, Pakistan \\ 2Institute of Space Technology, 75270, Karachi, Pakistan
}

\section{A R T I C L E I N F O}

\section{Article history:}

Received 21 February 2017

Received in revised form

7 June 2017

Accepted 8 June 2017

\section{Keywords:}

Generalized Burger's model

Porosity

Magnetic field

Analytical solutions and rheological parameters

\begin{abstract}
A B S T R A C T
This analysis is devoted to investigate analytical solutions of magnetohydrodynamics (MHD) generalized Burger's fluid embedded with porous medium as a sum of Newtonian and non-Newtonian forms. The solutions are investigated for velocity field and shear stress and governing partial differential equations have been solved via the integral transforms. The solutions for velocity field and shear stress have been expressed into compact form i-e in terms of series form. The general solutions also satisfy initial and boundary condition and particularized for special cases along with sum of Newtonian and non-Newtonian forms. The impacts of permeability (porosity), magnetism and several rheological parameters have been analyzed for fluid flows by portraying graphical illustrations. The graphs are depicted via latest software namely Mathematica and Mathcad (16) packages.
\end{abstract}

(C) 2017 The Authors. Published by IASE. This is an open access article under the CC BY-NC-ND license (http://creativecommons.org/licenses/by-nc-nd/4.0/).

\section{Introduction}

The linearized relationship between rate of strain and stress cannot be characterized by nonNewtonian fluids because of the involvement of stress tensor and relaxation which convert viscoelastic fluids into highly complicated and nonlinear in contrast with Newtonian fluids. NonNewtonian fluids even have gotten considerable attention because of their useful applications in industries and engineering. For instance, emulsion, heavy oils, chyme, blood and polymer solutions etc. Such fluids are modeled by their proposed constitutive relationships with symmetries. The description of these fluids are modeled to characterize viscoelastic behavior of flows as Maxwell, Oldrord-B, Burger models characterize small relaxation phenomenon, retardation times, rheological properties respectively (Zhaosheng and Jianzhong, 1998; Rajagopal, 1982; Zhang et al., 2007; Tan and Masuoka 2007; Abro, 2016; Fetecau et al., 2008; Abro and Shaikh, 2015). Furthermore, the main significance of Burger's model is to describe the cheese in food products and asphalts in geomechanics. Also, the characteristics of motion of

\footnotetext{
* Corresponding Author.

Email Address: kashif.abro@faculty.muet.edu.pk (K. A. Abro) https://doi.org/10.21833/ijaas.2017.07.012

2313-626X/C) 2017 The Authors. Published by IASE.

This is an open access article under the CC BY-NC-ND license

(http://creativecommons.org/licenses/by-nc-nd/4.0/)
}

the earths' mantle, asphalt concrete, asphalt, Olivine rocks, geological structures and propagation of seismic waves in the interior of the earth can be described by this model (Murali Krishnan and Rajagopal, 2004). Due to diverse applications, Burgers model has received much attention. In continuation we present few references for the study of Burger model (Jamil, 2012; Tong, 2010; Jamil and Fetecau, 2010; Khan et al., 2006; Hayat et al., 2010). The analysis for electrical conducting fluid flows is termed as magnetohydrodynamics has diverted attentions of many scientists and researchers due to its vast application in science and engineering. In geophysics and astrophysics, magnetohydrodynamics is useful to study solar plasma, terrestrial cores, cosmical fluid dynamics, structure of rotating magnetic stars, and stellar structures. The significance of magnetohydrodynamics in science and engineering, particularly in industrial applications of magnetohydrodynamics lies in extraction of geothermal energy, crystal growth in the field of semiconductors, magnetohydrodynamics (MHD) pumps, metallurgical and material processes and nuclear reactors (Hsiao, 2011; Zheng et al., 2012; Ahmad and Nazar, 2010). Obtaining the analytical solutions in presence of porous medium and magnetohydrodynamics is not an easy task, that why solutions in literatures are very rare. Having these complexities several scholars are busy in order to contribute in this phenomenon (presence and 
absence of porous medium and MHD). In our cognizance, some valuable contribution has been made by Khan et al. (2011) in which they studied magnetohydrodynamics flow of second grade fluid in porous medium for closed form solution. Hussain et al. (2010) has investigated second grade fluid in porous medium under oscillatory flows. Khan et al. (2012) has analyzed effects of thermal diffusion and ramped wall temperature for magnetohydrodynamics free convection flow in a porous medium. Furthermore few interested studies especially on MHD have been made by several researcher, we include here references in (Hayat et al., 2008a, 2008b; Abbasbandy et al., 2014; Freidoonimehr et al., 2015; Rashidi and Erfani, 2012; Rashidi et al., 2014). By the inspirations and motivations of above mentioned work, this analysis is devoted to investigate analytical solutions of MHD generalized Burger's fluid embedded with porous medium as a sum of Newtonian and non-Newtonian forms. The solutions are investigated for velocity field and shear stress and governing partial differential equations have been solved via the integral transformational techniques. The solutions for velocity field and shear stress have been expressed into compact form i-e in terms of series form. The general solutions also satisfy initial and boundary condition and particularized for special cases along with sum of Newtonian and nonNewtonian forms. The impacts of various rheological parameters have been analyzed for six models namely (i) Generalized Burger model, (ii) Burger model, (iii) Oldroyd-B model, (iv) Maxwell model, (v) Second Grade model and (vi) Newtonian model. These models are also discussed with and without porous medium and magnetohydrodynamics effects on fluid flow.

\section{Modeling of governing equations}

The constitutive equations for an incompressible generalized Burgers' fluid are (Jamil, 2012; Tong, 2010; Jamil and Fetecau, 2010; Khan et al., 2006) (Eq. 1)

$T=-p \mathrm{I}+S, \quad \mu\left(A_{1}+\lambda_{4} \frac{\delta^{2} A_{1}}{\delta t^{2}}+\lambda_{3} \frac{\delta A_{1}}{\delta t}\right)=S+\lambda_{2} \frac{\delta^{2} S}{\delta t^{2}}+$

$\lambda_{1} \frac{\delta S}{\delta t}$

where, $p$ the pressure, I the identity tensor, $\mathrm{S}$ is the extra-stress tensor, $\mu$ the dynamic viscosity, $A=$ $L+L^{T}$ is the first Rivlin-Ericksen tensor, $L$ denotes velocity gradient, $\lambda_{1}$ and $\lambda_{3}\left(<\lambda_{1}\right)$ are relaxation and retardation time, $\lambda_{2}$ and, $\lambda_{4}$ are new material parameters of the generalized Burgers' fluid and $\frac{\delta}{\delta t}$ denotes the upper convected time derivative defined as (Eq. 2)

$\frac{\delta^{2} S}{\delta t^{2}}=\frac{\delta}{\delta t}\left(\frac{\delta S}{\delta t}\right), \quad \frac{\delta S}{\delta t}=\frac{d S}{d t}-L S-S L^{T}$, $\frac{d}{d t}$ represents material time derivative. The unsteady flow of incompressible fluid is governed by (Eqs. 3 and 4):

$\operatorname{div} \boldsymbol{V}=0$

$\rho \frac{d V}{d t}=\operatorname{div} T-\sigma B_{0}^{2} V+R$,

where, $\rho$ is the density of the fluid, $V$ is the velocity, $\sigma$ the electrical conductivity of the fluid, $B_{0}$ the applied magnetic field's magnitude and $R$ denotes the Darcy's resistance. For the problem under consideration we assume a velocity field an extrastress tensor of the form (Eq. 5)

$V=V(y, t)=w(y, t) i, S=S(y, t)$

where $i$ is the unit vector along the x-co-ordinate direction. Introducing Eq. 5 in Eq. 1 and keeping initial conditions in mind (Eq. 6)

$S(y, 0)=\frac{\partial S(y, 0)}{\partial t}=0$,

yields $\tau_{z z}=\tau_{x z}=\tau_{y y}=\tau_{y z}=0$, and (Eq. 7)

$\mu\left(\lambda_{4} \frac{\partial^{2}}{\partial t^{2}}+\lambda_{3} \frac{\partial}{\partial t}+1\right) \frac{\partial w}{\partial y}=\left(\lambda_{2} \frac{\partial^{2}}{\partial t^{2}}+\lambda_{1} \frac{\partial}{\partial t}+1\right) S_{x y}$,

in which tangential stress is $S_{x y}$. With reference (Tan and Masuoka, 2007), the generalized Burger's fluid has relation for $R$ is

$-\frac{\mu \emptyset}{k}\left(\lambda_{4} \frac{\partial^{2}}{\partial t^{2}}+\lambda_{3} \frac{\partial}{\partial t}+1\right) \boldsymbol{V}(y, t)=\boldsymbol{R}\left(\lambda_{2} \frac{\partial^{2}}{\partial t^{2}}+\lambda_{1} \frac{\partial}{\partial t}+1\right)$,

where, $k$ is the permeability of the porous medium and $\varnothing$ is the porosity. Assuming that there is no pressure gradient in the flow direction and introducing Eq. 5 into Eq. 4 keeping in mind Eqs. 7, 8, we obtain following governing equations as (Jamil, 2012; Tong, 2010; Jamil and Fetecau, 2010; Khan et al., 2006; Hayat et al., 2010) (Eqs. 9 and 10)

$\left(\lambda_{2} \frac{\partial^{2}}{\partial t^{2}}+\lambda_{1} \frac{\partial}{\partial t}+1\right) \frac{\partial w(y, t)}{\partial t}=v\left(\lambda_{4} \frac{\partial^{2}}{\partial t^{2}}+\lambda_{3} \frac{\partial}{\partial t}+1\right) \frac{\partial^{2} w(y, t)}{\partial y^{2}}-$ $B\left(\lambda_{2} \frac{\partial^{2}}{\partial t^{2}}+\lambda_{1} \frac{\partial}{\partial t}+1\right) w(y, t)-\Phi\left(\lambda_{4} \frac{\partial^{2}}{\partial t^{2}}+\lambda_{3} \frac{\partial}{\partial t}+\right.$

1) $w(y, t)$

$\left(\lambda_{2} \frac{\partial^{2}}{\partial t^{2}}+\lambda_{1} \frac{\partial}{\partial t}+1\right) \tau(y, t)=\mu\left(\lambda_{4} \frac{\partial^{2}}{\partial t^{2}}+\lambda_{3} \frac{\partial}{\partial t}+1\right) \frac{\partial w(y, t)}{\partial t}$,

where, $=\frac{\mu}{\rho}, B=\frac{\sigma B_{0}}{\rho}, \Phi=\frac{\mu \emptyset}{k}$ are kinematic viscosity, applied magnetic field, porous medium of the fluid respectively.

\section{Statement of the problem}

We consider an incompressible and electrically conducting generalized Burgers fluid in porous medium occupying the space above a plate perpendicular to the $y$-axis. The plate is permeated under an influence of magnetic field $B_{0}$ normal to the flow in porous medium. For $t=0^{+}$the plate begins to accelerate in its own plane with velocity $w(0, t)=$ $U H(t) t^{p}$. Due to the shear, the fluid above the plate 
is gradually moved, while the governing equations are given by Eqs. 9 and 10 and corresponding initial, boundary and natural conditions are (Eqs. 11-13):

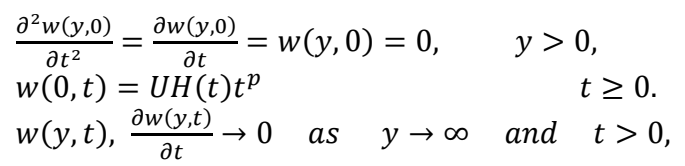

\section{Solution of the problem}

\subsection{Calculation of velocity field}

In order to explore the exact solution, we shall apply the Fourier sine transform to Eq. 9, and having in account the imposed conditions (11) and (12), we find that(Eq. 14)

$$
\begin{aligned}
& \left(\lambda_{2} \frac{\partial^{2}}{\partial t^{2}}+\lambda_{1} \frac{\partial}{\partial t}+1\right) \frac{\partial w_{s}(\xi, t)}{\partial t}-v\left(\lambda_{4} \frac{\partial^{2}}{\partial t^{2}}+\lambda_{3} \frac{\partial}{\partial t}+\right. \\
& \text { 1) }\left\{-\xi^{2} w_{S}(\xi, t)+\sqrt{\frac{2}{\pi}} w(0, t)\right\}+B\left(\lambda_{2} \frac{\partial^{2}}{\partial t^{2}}+\lambda_{1} \frac{\partial}{\partial t}+\right. \\
& \text { 1) } w_{s}(\xi, t)+\Phi\left(\lambda_{4} \frac{\partial^{2}}{\partial t^{2}}+\lambda_{3} \frac{\partial}{\partial t}+1\right) w_{S}(\xi, t)=0,
\end{aligned}
$$

where, $w_{s}(\xi, t)$ is the Fourier sine transform of $w(y, t)$ defined in Eq. 14 (Eq. 15)

$w_{S}(\xi, t)=\sqrt{\frac{2}{\pi}} \int_{0}^{\infty} w(y, t) \operatorname{Sin}(y \xi) d y$,

where the Fourier sine transform has to justify imposed conditions Eq. 11, (Eq. 16)

$\frac{\partial^{2} w_{s}(\xi, 0)}{\partial t^{2}}=\frac{\partial w_{s}(\xi, 0)}{\partial t}=w_{S}(\xi, 0)=0, \quad \xi>0$,

applying Laplace transform to Eq. 15 and keeping imposed conditions Eqs. 11 and 12, we obtain

$\bar{w}_{S}(\xi, s)=$
$\sqrt{\frac{2}{\pi}} \frac{U v \xi p !}{s^{p+1}}$ $\left[\frac{\left(\lambda_{4} s^{2}+\lambda_{3} s+1\right)}{\left[\lambda_{2} s^{3}+\left\{\lambda_{1}+\lambda_{2} B+\lambda_{4}\left(\Phi+v \xi^{2}\right)\right\} s^{2}+\left\{1+\lambda_{1} B+\lambda_{3}\left(\Phi+v \xi^{2}\right)\right\} s+\left(\Phi+B+v \xi^{2}\right)\right]}\right]$

in order to write the solutions as a sum of Newtonian and non-Newtonian Part $w_{N}(y, t)+w_{N o n}(y, t)$, we make $\lambda_{1}=\lambda_{2}=\lambda_{3}=\lambda_{4}=0$ in Eq. 17, we have expression for Newtonian part as (Eq. 18)

$\bar{w}_{S(N)}(\xi, s)=\sqrt{\frac{2}{\pi}} \frac{U v \xi p !}{s^{p+1}\left(s+\Phi+B+v \xi^{2}\right)}$,

substituting Eq. 18 into Eq. 17, we find balanced equation (Eq. 19)

$\bar{W}_{S}(\xi, s)=U \sqrt{\frac{2}{\pi}} \frac{v \xi p !}{\left(\Phi+B+v \xi^{2}\right)}\left\{\frac{1}{s^{p+1}}-\frac{1}{s^{p}\left(s+\Phi+B+v \xi^{2}\right)}\right\}+$
$U \sqrt{\frac{2}{\pi}} \frac{v \xi p !}{\lambda_{2}} \times\left[\frac{\left(\lambda_{4}-\lambda_{3}\right) s^{2}+\left\{\left(\lambda_{4}-\lambda_{2}\right) B+\left(\lambda_{3}-\lambda_{1}\right)\right\} s+\left(\lambda_{3} B-\lambda_{1}\right)}{s^{p}\left(s+\Phi+B+v \xi^{2}\right)\left(s-s_{1}\right)\left(s-s_{2}\right)\left(s-s_{3}\right)}\right]$

where, $\left(s-s_{1}\right)\left(s-s_{2}\right)\left(s-s_{3}\right)$ are the roots of an algebraic equation

$\lambda_{2} s^{3}+\left\{\lambda_{1}+\lambda_{2} B+\lambda_{4}\left(\Phi+v \xi^{2}\right)\right\} s^{2}+\left\{1+\lambda_{1} B+\right.$

$\left.\lambda_{3}\left(\Phi+v \xi^{2}\right)\right\} s+\left(\Phi+B+v \xi^{2}\right)$.

Inverting Eq. 19 by means of Fourier sine transform, we get (Eq. 20)

$\bar{w}(y, s)=\frac{2 U v \xi p !}{\pi} \int_{0}^{\infty} \frac{\sin (y \xi)}{\left(\Phi+B+v \xi^{2}\right)}\left\{\frac{1}{s^{p+1}}-\frac{1}{s^{p}\left(\mathrm{~s}+\Phi+B+v \xi^{2}\right)}\right\} d \xi+$ $\frac{2 U v p !}{\pi \lambda_{2}} \int_{0}^{\infty} \xi \sin (y \xi) \times$

$\left[\frac{\left(\lambda_{4}-\lambda_{3}\right) s^{2}+\left\{\left(\lambda_{4}-\lambda_{2}\right) B+\left(\lambda_{3}-\lambda_{1}\right)\right\} s+\left(\lambda_{3} B-\lambda_{1}\right)}{s^{p}\left(s+\Phi+B+v \xi^{2}\right)\left(s-s_{1}\right)\left(s-s_{2}\right)\left(s-s_{3}\right)}\right] d \xi$,

employing the fact of integral Eq. 21

$\int_{0}^{\infty} \frac{\xi \sin (\gamma \xi)}{\alpha^{2}+\xi^{2}} d \xi=\frac{\pi}{2} e^{-\alpha \gamma}, \quad \alpha>0$,

and using inverse Laplace transform in Eq. 20, we obtain convolution product as (Eq. 22)

$w(y, t)=U H(t) t^{p} \operatorname{Exp}\left(-y \sqrt{\frac{\Phi+B}{v}}\right)-\frac{2 U H(t) v p}{\pi} \int_{0}^{\infty} \frac{\xi \sin (y \xi)}{\left(\Phi+B+v \xi^{2}\right)} t^{p-1} * \operatorname{Exp}\left(-\Phi-B-v \xi^{2}\right) d \xi+\frac{2 U H(t) v p}{\pi \lambda_{2}} \times \int_{0}^{\infty} \xi \sin (y \xi) t^{p-1} *$ $\left[\begin{array}{c}\frac{\left(\lambda_{4}-\lambda_{3}\right) s_{1}^{2}+\left\{\left(\lambda_{4}-\lambda_{2}\right) B+\left(\lambda_{3}-\lambda_{1}\right)\right\} s_{1}+\left(\lambda_{3} B-\lambda_{1}\right) \operatorname{Exp}\left(s_{1}\right) t}{\left(s_{1}+\Phi+B+v \xi^{2}\right)\left(s_{1}-s_{2}\right)\left(s_{1}-s_{3}\right)} \\ +\frac{\left(\lambda_{4}-\lambda_{3}\right) s_{2}^{2}+\left\{\left(\lambda_{4}-\lambda_{2}\right) B+\left(\lambda_{3}-\lambda_{1}\right)\right\} s_{2}+\left(\lambda_{3} B-\lambda_{1}\right) \operatorname{Exp}\left(s_{2}\right) t}{\left(s_{2}+\Phi+B+v \xi^{2}\right)\left(s_{2}-s_{1}\right)\left(s_{2}-s_{3}\right)} \\ +\frac{\left(\lambda_{4}-\lambda_{3}\right) s_{3}^{2}+\left\{\left(\lambda_{4}-\lambda_{2}\right) B+\left(\lambda_{3}-\lambda_{1}\right)\right\} s_{3}+\left(\lambda_{3} B-\lambda_{1}\right) \operatorname{Exp}\left(s_{3}\right) t}{\left(s_{3}+\Phi+B+v \xi^{2}\right)\left(s_{3}-s_{1}\right)\left(s_{3}-s_{2}\right)} \\ +\frac{\left(\lambda_{4}-\lambda_{3}\right)\left(\Phi+B+v \xi^{2}\right)^{2}+\left\{\left(\lambda_{4}-\lambda_{2}\right) B+\left(\lambda_{3}-\lambda_{1}\right)\right\}\left(\Phi+B+v \xi^{2}\right)+\left(\lambda_{3} B-\lambda_{1}\right)}{\left(s_{1}+\Phi+B+v \xi^{2}\right)\left(s_{2}+\Phi+B+v \xi^{2}\right)\left(s_{3}+\Phi+B+v \xi^{2}\right)} \\ \times \operatorname{Exp}\left(-\Phi-B-v \xi^{2}\right) t^{(-B)}\end{array}\right] d \xi$,

finally velocity field is expressed in compact form in terms of series, (Eq. 23)

$$
\begin{aligned}
& w(y, t)=w_{N}(y, t)+\frac{2 U H(t) v p}{\pi \lambda_{2}} \int_{0}^{\infty} \int_{0}^{t} \xi \sin (y \xi)(t-z)^{p-1} \times \\
& {\left[\begin{array}{c}
\frac{\left(\lambda_{4}-\lambda_{3}\right)\left(\Phi+B+v \xi^{2}\right)^{2}+\left\{\left(\lambda_{4}-\lambda_{2}\right) B+\left(\lambda_{3}-\lambda_{1}\right)\right\}\left(\Phi+B+\nu \xi^{2}\right)+\left(\lambda_{3} B-\lambda_{1}\right)}{\left(s_{1}+\Phi+B+v \xi^{2}\right)\left(s_{2}+\Phi+B+\nu \xi^{2}\right)\left(s_{3}+\Phi+B+\nu \xi^{2}\right)} \\
\times e^{\left(-\Phi-B-v \xi^{2}\right) z}+\sum_{n=1}^{3} \frac{\left(\lambda_{4}-\lambda_{3}\right) s_{n}^{2}+\left\{\left(\lambda_{4}-\lambda_{2}\right) B+\left(\lambda_{3}-\lambda_{1}\right) s_{n}+\left(\lambda_{3} B-\lambda_{1}\right) e^{s_{n} z}\right.}{\left(s_{n}+\Phi+B+\nu \xi^{2}\right)\left(s_{n}-s_{(3-n) !}\right)\left(s_{n}-s_{4-(n-1) !}\right)}
\end{array}\right] d \xi d z .}
\end{aligned}
$$

where, $w_{N}(y, t)=U H(t) t^{p} \operatorname{Exp}\left(-y \sqrt{\frac{\Phi+B}{v}}\right)-\frac{2 U H(t) v p}{\pi} \int_{0}^{\infty} \int_{0}^{t} \frac{\xi \sin (y \xi)}{\left(\Phi+B+v \xi^{2}\right)}(t-z)^{p-1} \operatorname{Exp}\left(-\Phi-B-v \xi^{2}\right) z d \xi d z$ is the Newtonian part velocity field. 


\subsection{Calculation of shear stress}

For finding shear stress, we apply Laplace transform to Eq. 10, we find that (Eq. 24)

$\bar{\tau}(y, s)=\mu\left(\frac{\lambda_{4} q^{2}+\lambda_{3} q+1}{\lambda_{2} q^{2}+\lambda_{1} q+1}\right) \frac{\partial \bar{w}(y, s)}{\partial y}$,

in order to write the solutions as a sum of Newtonian and non-Newtonian Part $\tau_{N}(y, t)+\tau_{N o n}(y, t)$, we make $\lambda_{1}=\lambda_{2}=\lambda_{3}=\lambda_{4}=0$ in Eq. 24, we have expression for Newtonian part as (Eq. 25)

$\bar{\tau}_{(N)}(y, s)=\mu \frac{\partial \bar{w}(y, s)}{\partial y}$,

$\bar{\tau}(y, s)=-\frac{2 U \mu \nu p !}{\pi} \int_{0}^{\infty} \frac{\xi^{2} \cos (y \xi)}{\left(\Phi+B+v \xi^{2}\right)} \frac{1}{s^{p}\left(\mathrm{~s}+\Phi+B+v \xi^{2}\right)} d \xi-\frac{2 U \mu \nu p !}{\pi \lambda_{2}} \int_{0}^{\infty} \frac{\xi^{2} \cos (y \xi)}{\left(\Phi+B+v \xi^{2}\right)} \frac{1}{s^{p}} d \xi \times$

$$
\left[\begin{array}{c}
\frac{\left(\lambda_{4}-\lambda_{2}\right) s_{1}^{2}+\left\{\left(\lambda_{4}-\lambda_{2}\right) B+\left(\lambda_{3}-\lambda_{1}\right)\right\} s_{1}+\left(\lambda_{3}-\lambda_{1}\right) B}{\left(s_{1}+\Phi+B+v \xi^{2}\right)\left(s_{1}-s_{2}\right)\left(s_{1}-s_{3}\right)\left(s-s_{1}\right)} \\
+\frac{\left(\lambda_{4}-\lambda_{2}\right) s_{1}^{2}+\left\{\left(\lambda_{4}-\lambda_{2}\right) B+\left(\lambda_{3}-\lambda_{1}\right)\right\} s_{1}+\left(\lambda_{3}-\lambda_{1}\right) B}{\left(s_{2}+\Phi+B+v \xi^{2}\right)\left(s_{2}-s_{1}\right)\left(s_{2}-s_{3}\right)\left(s-s_{2}\right)} \\
+\frac{\left(\lambda_{4}-\lambda_{2}\right) s_{1}^{2}+\left\{\left(\lambda_{4}-\lambda_{2}\right) B+\left(\lambda_{3}-\lambda_{1}\right)\right\} s_{1}+\left(\lambda_{3}-\lambda_{1}\right) B}{\left(s_{2}+\Phi+B+v \xi^{2}\right)\left(s_{3}-s_{1}\right)\left(s_{3}-s_{2}\right)\left(s-s_{3}\right)} \\
+\frac{\left(\lambda_{4}-\lambda_{2}\right)\left(\Phi+B+v \xi^{2}\right)^{2}+\left\{\left(\lambda_{4}-\lambda_{2}\right) B+\left(\lambda_{3}-\lambda_{1}\right)\right\}\left(\Phi+B+v \xi^{2}\right)+\left(\lambda_{3}-\lambda_{1}\right) B}{\left(s_{1}+\Phi+B+v \xi^{2}\right)\left(s_{2}+\Phi+B+v \xi^{2}\right)\left(s_{3}+\Phi+B+v \xi^{2}\right)\left(s+\Phi+B+v \xi^{2}\right)}
\end{array}\right.
$$

finally applying inverse Laplace transform to Eq. 27, we expressed shear stress in compact form in terms of series,

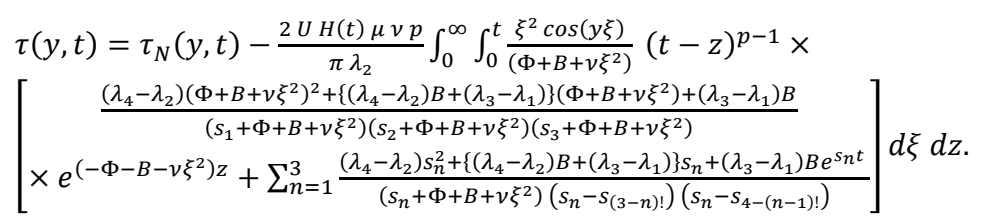

where,

$\tau_{N}(y, t)=-\frac{2 U H(t) \mu v p}{\pi} \int_{0}^{\infty} \int_{0}^{t} \frac{\xi^{2} \cos (y \xi)}{\left(\Phi+B+v \xi^{2}\right)}(t-z)^{p-1} \operatorname{Exp}\left(-\Phi-B-v \xi^{2}\right) z d \xi d z$.

is the Newtonian part of shear stress.

\section{Limiting case}

Employing same procedure we obtain here some special cases of general solutions of velocity field and corresponding shear stress.

$$
w_{B}(y, t)=w_{N}(y, t)-\frac{2 U H(t) v p}{\pi \lambda_{2}} \int_{0}^{\infty} \int_{0}^{t} \xi \sin (y \xi)(t-z)^{p-1} \times\left[\begin{array}{r}
\frac{\lambda_{3} s_{4}^{2}+\left(\lambda_{3}-B \lambda_{2}-\lambda_{1}\right) s_{4}+\left(\lambda_{3} B-\lambda_{1}\right) e^{s_{4} z}}{\left(s_{4}+\Phi+B+v \xi^{2}\right)\left(s_{4}-s_{5}\right)\left(s_{4}-s_{6}\right)} \\
+\frac{\lambda_{3} s_{5}^{2}+\left(\lambda_{3}-B \lambda_{2}-\lambda_{1}\right) s_{5}+\left(\lambda_{3} B-\lambda_{1}\right) e^{s_{5} z}}{\left(s_{5}+\Phi+B+v \xi^{2}\right)\left(s_{5}-s_{4}\right)\left(s_{5}-s_{6}\right)} \\
+\frac{\lambda_{3} s_{6}^{2}+\left(\lambda_{3}-B \lambda_{2}-\lambda_{1}\right) s_{6}+\left(\lambda_{3} B-\lambda_{1}\right) e^{s_{6} z}}{\left(s_{6}+\Phi+B+v \xi^{2}\right)\left(s_{6}-s_{4}\right)\left(s_{6}-s_{5}\right)} \\
\frac{\lambda_{3}\left(\Phi+B+v \xi^{2}\right)^{2}-\left\{\lambda_{3}-\lambda_{2} B-\lambda_{1}\right\}\left(\Phi+B+v \xi^{2}\right)+\left(\lambda_{3} B-\lambda_{1}\right)}{\left(s_{1}+\Phi+B+v \xi^{2}\right)\left(s_{2}+\Phi+B+v \xi^{2}\right)\left(s_{3}+\Phi+B+v \xi^{2}\right)} e^{\left(-\Phi-B-v \xi^{2}\right) z}
\end{array}\right]
$$

and corresponding shear stress

$$
\left.\begin{array}{c}
\tau(y, t)=\tau_{N}(y, t)-\frac{2 U H(t) \mu v p}{\pi \lambda_{2}} \int_{0}^{\infty} \int_{0}^{t} \frac{\xi^{2} \cos (y \xi)}{\left(\Phi+B+v \xi^{2}\right)}(t-z)^{p-1} \\
\frac{\lambda_{2} s_{4}^{2}-\left(\lambda_{1}+\lambda_{2} B-\lambda_{3}\right) s_{4}+\left(\lambda_{3}-\lambda_{1}\right) B \operatorname{Exp}\left(s_{4} z\right)}{\left(s_{4}+\Phi+B+v \xi^{2}\right)\left(s_{4}-s_{5}\right)\left(s_{4}-s_{6}\right)} \\
+\frac{\lambda_{2} s_{5}^{2}-\left(\lambda_{1}+\lambda_{2} B-\lambda_{3}\right) s_{5}+\left(\lambda_{3}-\lambda_{1}\right) B \operatorname{Exp}\left(s_{5} z\right)}{\left(s_{5}+\Phi+B+v \xi^{2}\right)\left(s_{5}-s_{4}\right)\left(s_{5}-s_{6}\right)} \\
+\frac{\lambda_{2} s_{6}^{2}-\left(\lambda_{1}+\lambda_{2} B-\lambda_{3}\right) s_{6}+\left(\lambda_{3}-\lambda_{1}\right) B \operatorname{Exp}\left(s_{6} z\right)}{\left(s_{6}+\Phi+B+v \xi^{2}\right)\left(s_{6}-s_{4}\right)\left(s_{6}-s_{5}\right)} \\
+\frac{\lambda_{2}\left(\Phi+B+v \xi^{2}\right)^{2}-\left(\lambda_{3}-\lambda_{2} B+\lambda_{1}\right)\left(\Phi+B+v \xi^{2}\right)+\left(\lambda_{3}-\lambda_{1}\right) B}{\left(s_{4}+\Phi+B+v \xi^{2}\right)\left(s_{5}+\Phi+B+v \xi^{2}\right)\left(s_{6}+\Phi+B+v \xi^{2}\right)} e^{\left(-\Phi-B-v \xi^{2}\right) z}
\end{array}\right] d \xi d z
$$


$\left(s-s_{4}\right)\left(s-s_{5}\right)\left(s-s_{6}\right)$ are the roots of an algebraic equation:

$$
\begin{gathered}
\lambda_{2} s^{3}+\left(\lambda_{1}+\lambda_{2} B\right) s^{2}+\left\{1+\lambda_{1} B+\lambda_{3}\left(\Phi+v \xi^{2}\right)\right\} s \\
+\left(\Phi+B+v \xi^{2}\right) .
\end{gathered}
$$

\subsection{Solutions of Oldroyd-B fluid}

Letting $\lambda_{4}=\lambda_{2}=0$ in Eqs. 23 and 28, we obtain the velocity field and shear stress of Oldrord-B fluid $w_{O B}(y, t)=w_{N}(y, t)+\frac{2 U H(t) v p}{\pi \lambda_{1}} \int_{0}^{\infty} \int_{0}^{t} \xi \sin (y \xi)(t-$ $z)^{p-1} \times\left[\begin{array}{c}\frac{\left(\lambda_{1}-\lambda_{3}\right)\left(s_{7}+B\right) e^{s_{7} z}}{\left(s_{7}+\Phi+B+\nu \xi^{2}\right)\left(s_{7}-s_{8}\right)}+\frac{\left(\lambda_{1}-\lambda_{3}\right)\left(s_{8}+B\right) e^{s_{8} z}}{\left(s_{8}+\Phi+B+\nu \xi^{2}\right)\left(s_{8}-s_{7}\right)} \\ +\frac{\left(\lambda_{1}-\lambda_{3}\right)\left(1+\Phi+B+\nu \xi^{2}\right)}{\left(s_{7}+\Phi+B+\nu \xi^{2}\right)\left(s_{8}+\Phi+B+\nu \xi^{2}\right)} e^{\left(-\Phi-B-\nu \xi^{2}\right) z}\end{array}\right] d \xi d z$,

and corresponding shear stress

$\tau_{O B}(y, t)=\tau_{N}(y, t)-\frac{2 U H(t) \mu v p}{\pi \lambda_{1}} \int_{0}^{\infty} \int_{0}^{t} \frac{\xi^{2} \cos (y \xi)}{\left(\Phi+B+v \xi^{2}\right)}(t-$ $z)^{p-1} \times$ $\left[\begin{array}{c}\frac{\left(\lambda_{1}-\lambda_{3}\right)\left(s_{7}+\lambda_{3} v \xi^{2}+B\right) e^{s_{7} z}}{\left(s_{7}+\Phi+B+v \xi^{2}\right)\left(s_{7}-s_{8}\right)}+\frac{\left(\lambda_{1}-\lambda_{3}\right)\left(s_{8}+\lambda_{3} v \xi^{2}+B\right) e^{s_{8} z}}{\left(s_{8}+\Phi+B+v \xi^{2}\right)\left(s_{8}-s_{7}\right)} \\ +\frac{\left(\lambda_{1}-\lambda_{3}\right)\left(\lambda_{3} v \xi^{2}+\Phi+2 B+v \xi^{2}\right)}{\left(s_{7}+\Phi+B+v \xi^{2}\right)\left(s_{8}+\Phi+B+v \xi^{2}\right)} e^{\left(-\Phi-B-v \xi^{2}\right) z}\end{array}\right] d \xi d z$

$\left(s-s_{7}\right)\left(s-s_{8}\right)$ are the roots of an algebraic equation:

$\lambda_{1} s^{2}+\left\{1+\lambda_{1} B+\lambda_{3}\left(\Phi+v \xi^{2}\right)\right\} s+\left(\Phi+B+v \xi^{2}\right)$.

\subsection{Solutions of Maxwell fluid}

Letting $\lambda_{4}=\lambda_{3}=\lambda_{2}=0$ in Eqs. 23 and 28, we obtain the velocity field and shear stress of Maxwell fluid

$w_{M}(y, t)=w_{N}(y, t)+\frac{2 U H(t) v p}{\pi \lambda_{1}} \int_{0}^{\infty} \int_{0}^{t} \xi \sin (y \xi)(t-$
$z)^{p-1} \times\left[\begin{array}{c}\frac{\lambda_{1}\left(s_{9}+B\right) e^{s_{9} z}}{\left(s_{9}+\Phi+B+v \xi^{2}\right)\left(s_{9}-s_{10}\right)}+\frac{\lambda_{1}\left(s_{10}+B\right) e^{s_{10} z}}{\left(s_{10}+\Phi+B+v \xi^{2}\right)\left(s_{10}-s_{9}\right)} \\ +\frac{\lambda_{1}\left(1+\Phi+B+v \xi^{2}\right)}{\left(s_{9}+\Phi+B+v \xi^{2}\right)\left(s_{10}+\Phi+B+v \xi^{2}\right)} e^{\left(-\Phi-B-v \xi^{2}\right) z}\end{array}\right] d \xi d z$,

and corresponding shear stress

$$
\begin{aligned}
& \tau_{M}(y, t)=\tau_{N}(y, t)-\frac{2 U H(t) \mu v p}{\pi \lambda_{1}} \int_{0}^{\infty} \int_{0}^{t} \frac{\xi^{2} \cos (y \xi)}{\left(\Phi+B+v \xi^{2}\right)}(t- \\
& z)^{p-1} \times\left[\begin{array}{c}
\frac{\lambda_{1}\left(s_{9}+B\right) e^{s_{9} z}}{\left(s_{9}+\Phi+B+v \xi^{2}\right)\left(s_{9}-s_{10}\right)}+\frac{\lambda_{1}\left(s_{10}+\lambda_{3} \nu \xi^{2}+B\right) e^{s_{10} z}}{\left(s_{10}+\Phi+B+v \xi^{2}\right)\left(s_{10}-s_{9}\right)} \\
+\frac{\lambda_{1}\left(\Phi+2 B+v \xi^{2}\right)}{\left(s_{9}+\Phi+B+v \xi^{2}\right)\left(s_{10}+\Phi+B+v \xi^{2}\right)} e^{\left(-\Phi-B-v \xi^{2}\right) z}
\end{array}\right] d \xi d z,
\end{aligned}
$$

$\left(s-s_{9}\right)\left(s-s_{10}\right)$ are the roots of an algebraic equation:

$\lambda_{1} s^{2}+\left(1+\lambda_{1} B\right) s+\left(\Phi+B+v \xi^{2}\right)$.

\subsection{Solutions of second grade fluid}

Letting $\lambda_{4}=\lambda_{2}=\lambda_{1}=0$ in Eqs. 23 and 28, we obtain the velocity field and shear stress of Second Grade fluid
$w_{S G}(y, t)=w_{N}(y, t)+\frac{2 U H(t) v p}{\pi} \int_{0}^{\infty} \int_{0}^{t} \frac{\xi \sin (y \xi)}{\left(1+\lambda_{3} \Phi+\lambda_{3} v \xi^{2}\right)}(t-$ z) ${ }^{p-1} \times$

$\left[\frac{\lambda_{3}\left(\Phi+v \xi^{2}\right) e^{\left(\frac{\Phi+B+v \xi^{2}}{1+\lambda_{3} \Phi+\lambda_{3} v \xi^{2}}\right) z}}{\left(\frac{\Phi+B+v \xi^{2}}{1+\lambda_{3} \Phi+\lambda_{3} v \xi^{2}}-\left(\Phi+B+v \xi^{2}\right)\right)}-\frac{\lambda_{3}\left(\Phi+v \xi^{2}\right) e^{\left(-\Phi-B-v \xi^{2}\right) z}}{\left(\frac{\Phi+B+v \xi^{2}}{1+\lambda_{3} \Phi+\lambda_{3} v \xi^{2}}-\left(\Phi+B+v \xi^{2}\right)\right)}\right] d \xi d z$

and corresponding shear stress

$\tau_{S G}(y, t)=\tau_{N}(y, t)+\frac{2 U H(t) \mu v p}{\pi} \int_{0}^{\infty} \int_{0}^{t} \frac{\xi^{2} \cos (y \xi)}{\left(\Phi+B+v \xi^{2}\right)}(t-$

$z)^{p-1} \times\left[\left(\frac{\Phi+B+v \xi^{2}}{1+\lambda_{3} \Phi+\lambda_{3} v \xi^{2}}\right) \lambda_{3}\left(2-\lambda_{3}\right) e^{-z}-\right.$

$\left.e^{\left(\frac{\Phi+B+v \xi^{2}}{1+\lambda_{3} \Phi+\lambda_{3} v \xi^{2}}\right) z}\right] d \xi d z$.

Also, solutions for Newtonian fluid when $\lambda_{1}=0$ in Eqs. 33 and 34 can be easily be retrieved in similar manners which are known in literature (Jamil, 2012).

\section{Concluding remarks}

In this portion is analyzed for the significance of permeability (porosity), magnetism and several rheological parameters, material parameters on the fluid flow due to accelerating plate for magnetohydrodynamics generalized Burger's fluid embedded with porous medium as a sum of Newtonian and non-Newtonian forms. In order to illustrate the differences and similarities among various graphs for relevant physical aspects, we have used different numerical values for instance, time, permeability, porosity, magnetic parameter, viscosity, non-zero constant, density, kinematic viscosity, relaxation time, retardation phenomenon, material and rheological parameters etc. However the major findings/outcomes are listed below:

1. The general solutions for velocity field and shear stress have been expressed into compact form i-e in terms of series form satisfying initial, boundary and natural conditions as well. These solutions are obtained employing four translations of integral transforms which are (i) Fourier Sine transform, (ii) Laplace transform, (iii) Inverse Fourier Sine transform and (iv) Inverse Laplace transform. The translation from (i) to (iv) are applied according to governing partial differential equation and usual initial and boundary conditions.

2. The influence of time parameter $t$ is depicted in Fig. 1 in which as time increases the both velocity and shear stress profiles are decreasing function in absolute values.

3. Fig. 2 is plotted for the rheology of viscosity $v$ of the fluid in which the elastic behavior of fluid has a tendency to enhance the profiles of velocity and shear stress generated by motion of accelerating plate.

4. Figs. 3, 4, 5, and 6 have been drawn for showing the influence on material parameters $\left(\lambda_{1}, \lambda_{2}, \lambda_{3}\right.$ 
and $\lambda_{4}$ ), by considering nearer and smaller values of $\lambda_{1}$ and $\lambda_{2}$ have similar and identical behavior of fluid flow as expected. It is worth pointed out that $\lambda_{1}$ and $\lambda_{3}$ relaxation and retardation phenomenon respectively have quietly contradictory effects on fluid flow for both profiles velocity as well as shear stress.

5. Fig. 7 is presents the profile of velocity and shear stress in which various extreme small values are taken for magnetic field $B$, it is clearly seen that it is a Lorentz force which resist the fluid flow. This is due to the fact that magnetic field B is applied in transverse direction.

6. The effects of permeability $K$ and porosity $\Phi$ on the fluid motion are depicted in Fig. 8 which has qualitatively scattering behavior on the fluid motion.

7. Fig. 9 is drawn to give variations for the behavior on the fluid motion in presence of magnetic field as well as porosity. In which the velocity field has squeezed motion of fluid as compared with profile of shear stress.

8. Figs. 10 and 11 display the variation in presence of porosity and magnetic field respectively for six models namely (i) Generalized Burger model, (ii) Burger model, (iii) Oldroyd-B model, (iv) Maxwell model, (v) Second Grade model and (vi) Newtonian model, in which generalized Burger's model is swiftest and Newtonian model has slowest behavior on motion either the presence of porosity and magnetic field or not.

(b)

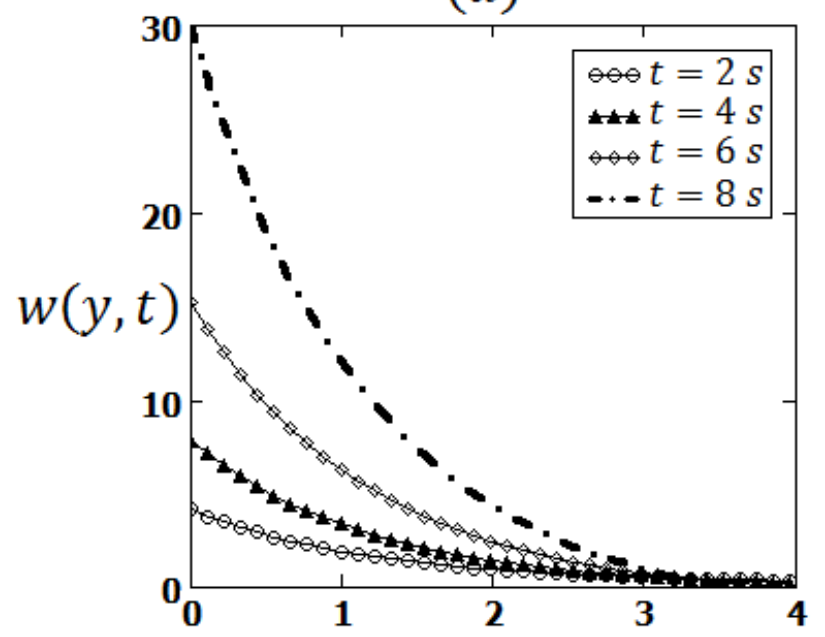

Fig. 1: Profile of velocity fields and shear stress for t at $U=2, v=1, \mu=2.5, \lambda_{1}=2, \lambda_{2}=7, \lambda_{3}=5, \lambda_{4}=4, p=2, B=$ $1.5, \Phi=2.74$ (a)

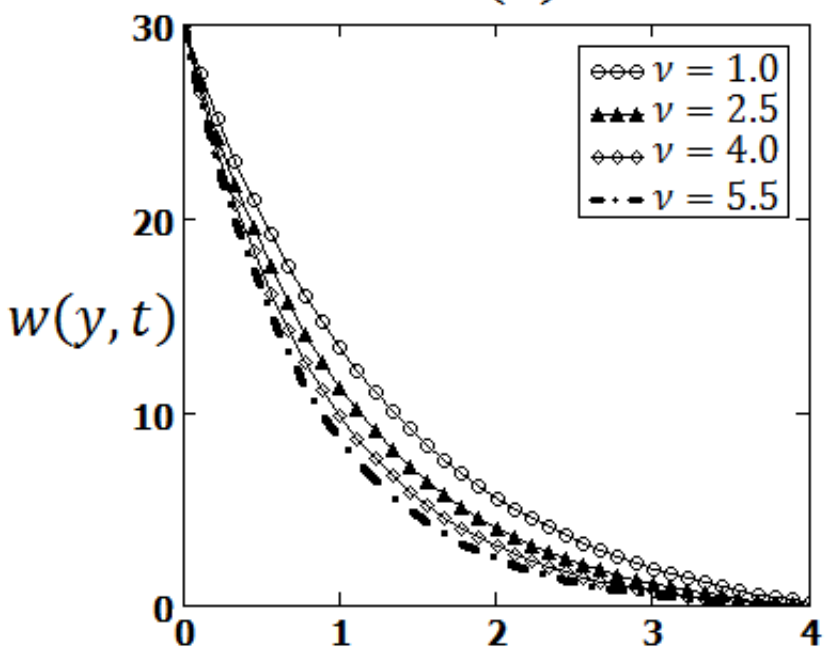

(b)

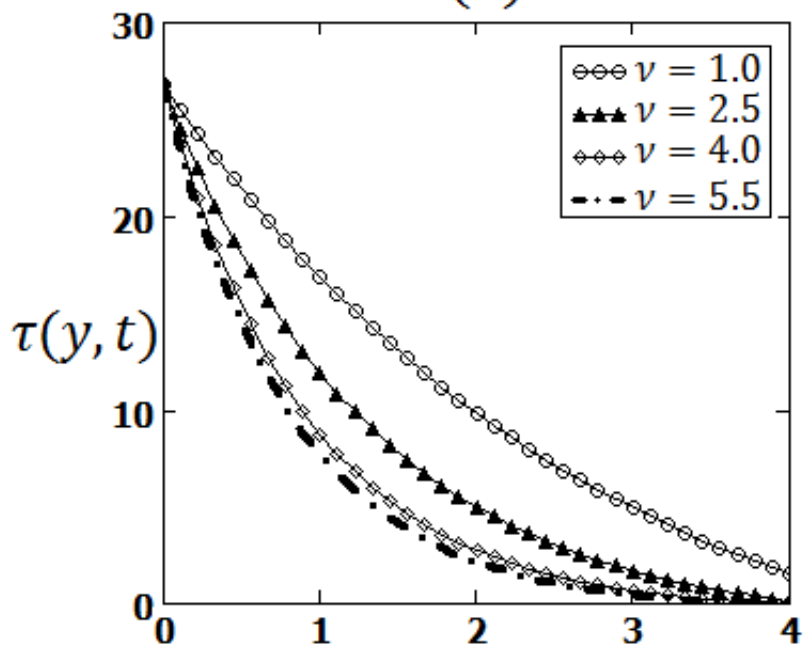

Fig. 2: Profile of velocity fields and shear stress for $v$ at $U=1, t=3 S, \mu=1.3, \lambda_{1}=0.2, \lambda_{2}=0.25, \lambda_{3}=1.5, \lambda_{4}=2.25, p=$ $1, B=2, \Phi=16$ 
(a)

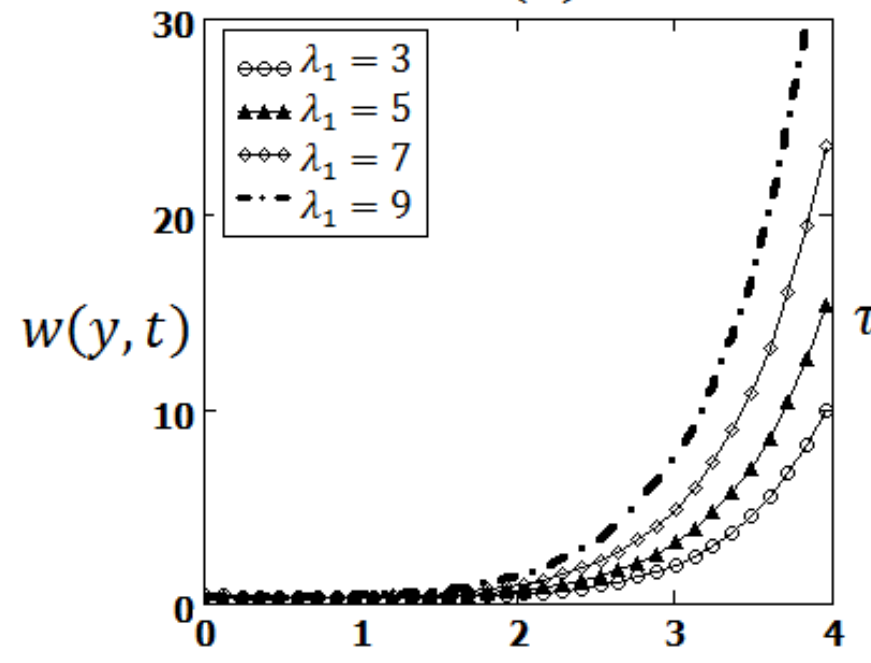

(b)

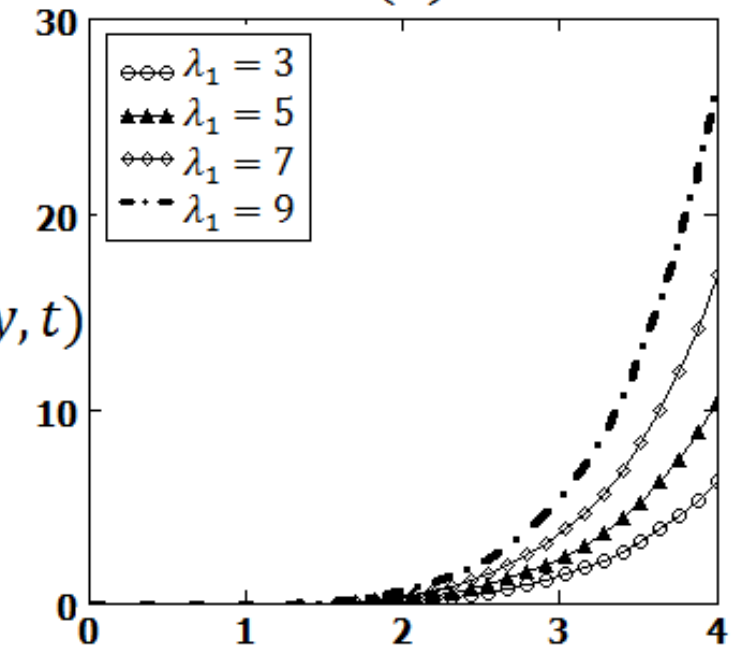

Fig. 3: Profile of velocity fields and shear stress for $\lambda_{1}$ at $U=1, t=3 S, v=3, \mu=1.3, \lambda_{2}=0.25, \lambda_{3}=1.5, \lambda_{4}=2.25, p=$

(a)

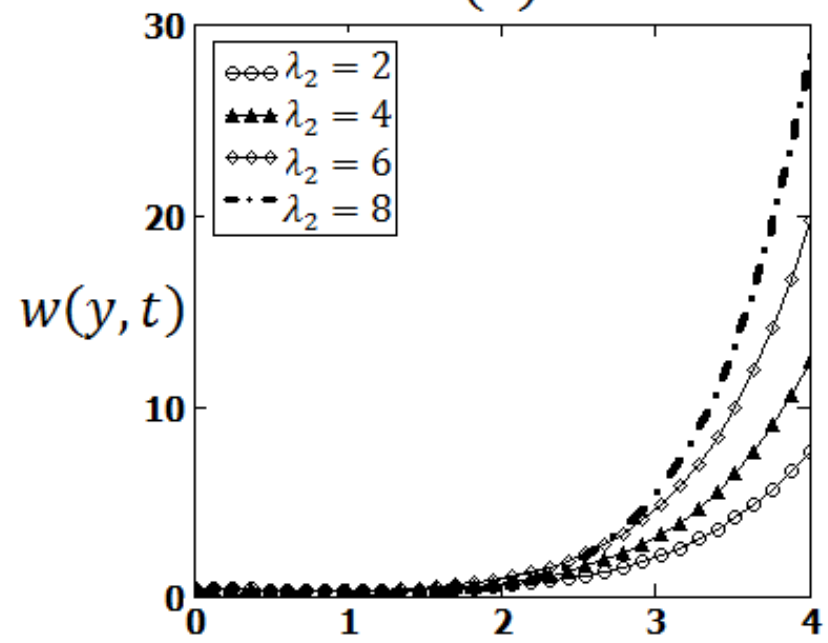

$1, B=2, \Phi=16$

(b)

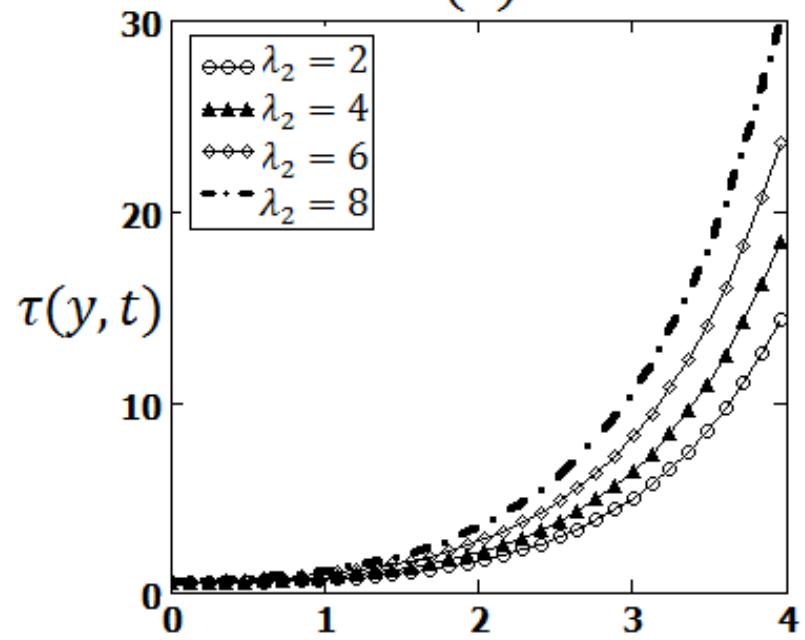

Fig. 4: Profile of velocity fields and shear stress for $\lambda_{2}$ at $U=2, t=2 S, v=2, \mu=2.9, \lambda_{1}=0.3, \lambda_{3}=1.2, \lambda_{4}=0.2, p=1$, $B=0.4, \Phi=8$

(a)

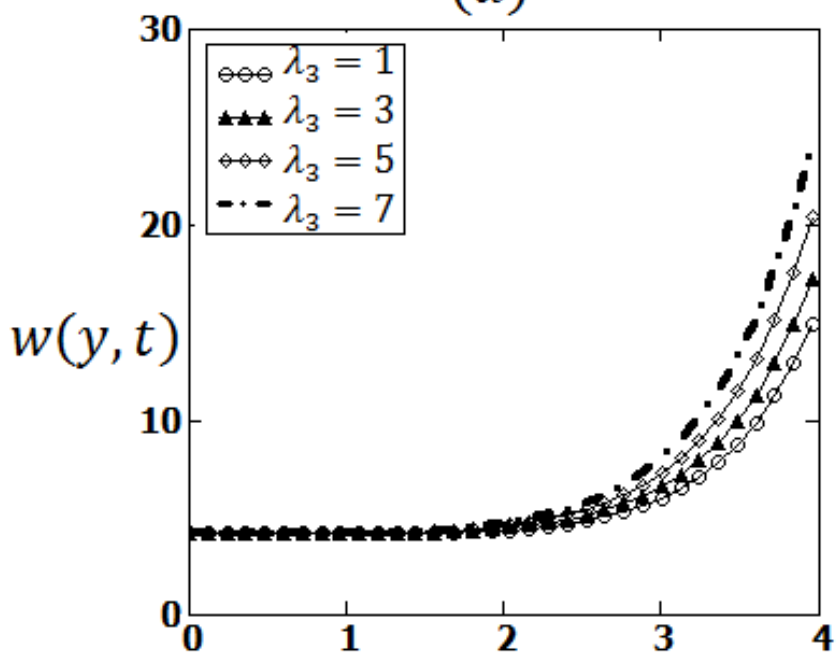

(b)

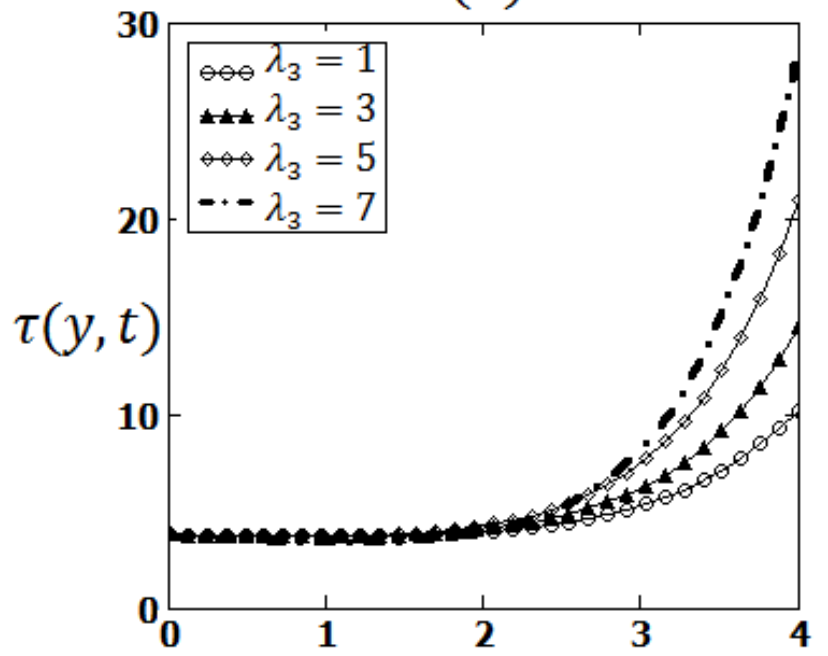

Fig. 5: Profile of velocity fields and shear stress for $\lambda_{3}$ at $U=2, t=2 S, v=1.5, \mu=7.2, \lambda_{1}=5, \lambda_{2}=1.2, \lambda_{4}=19, p=1$, $B=5, \Phi=3$ 
(a)

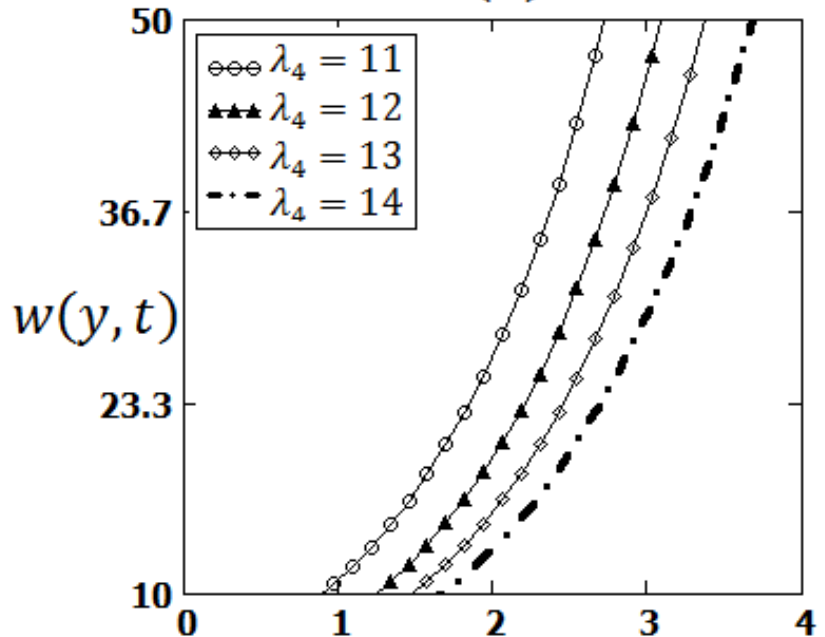

$(b)$

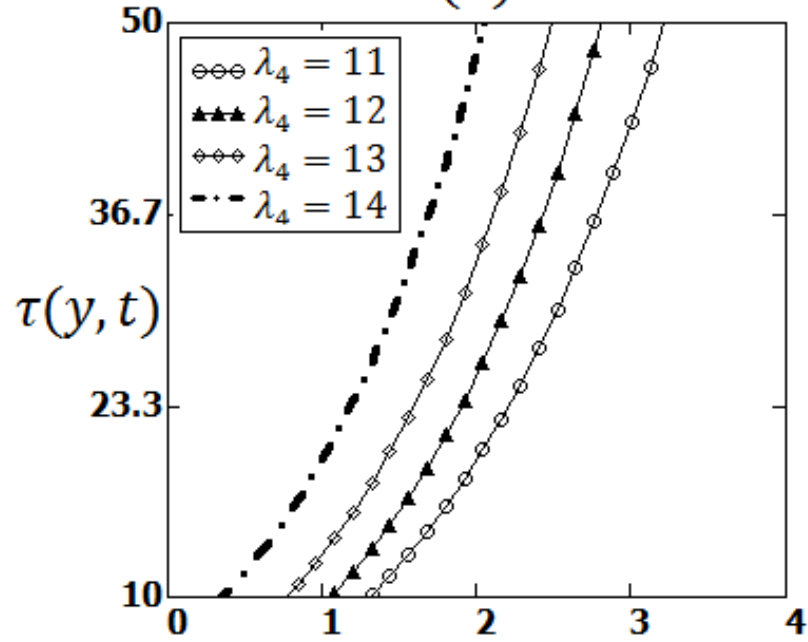

Fig. 6: Profile of velocity fields and shear stress for $\lambda_{4}$ at $U=5, t=3 S, v=6.25, \mu=7.2, \lambda_{1}=12, \lambda_{2}=33, \lambda_{3}=104, p=$

(a)

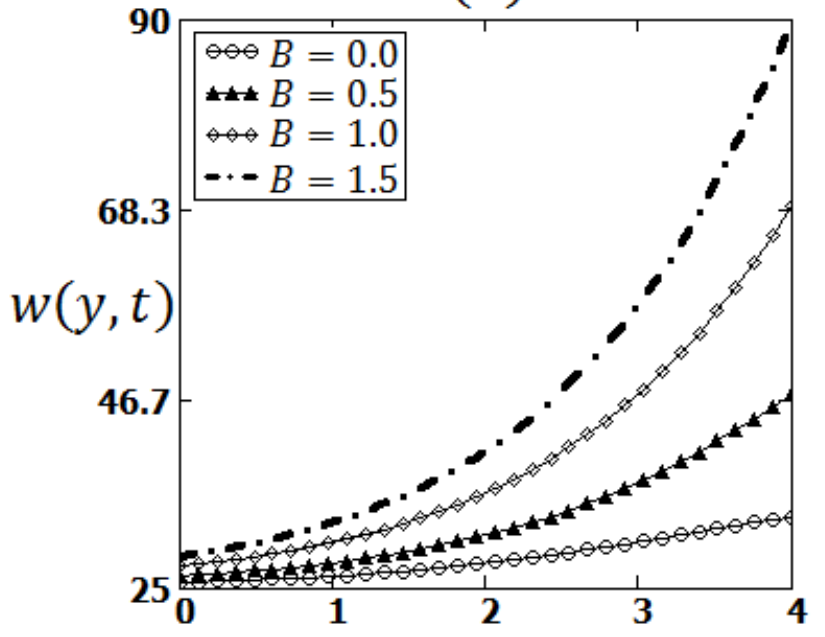

(b)

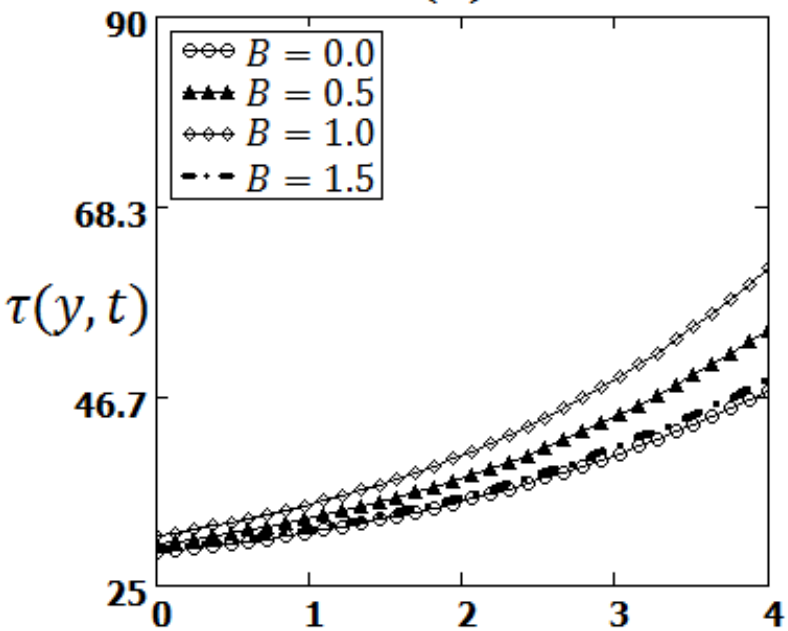

Fig. 7: Profile of velocity fields and shear stress for $B$ at $U=1, t=2 S, v=0.25, \mu=2.8, \lambda_{1}=1.2, \lambda_{2}=2.2, \lambda_{3}=0.5, \lambda_{4}=$ $0.2, p=1, \Phi=3.75$

(a)

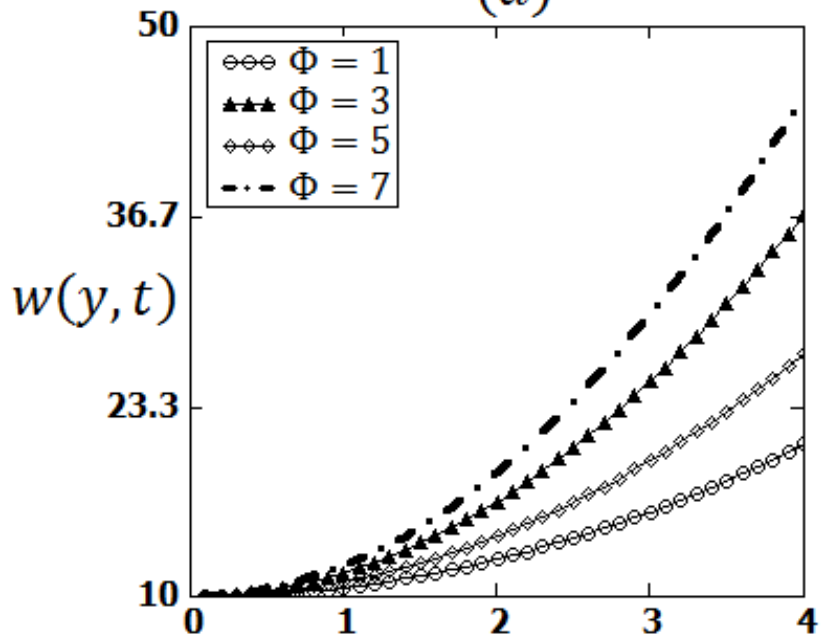

(b)

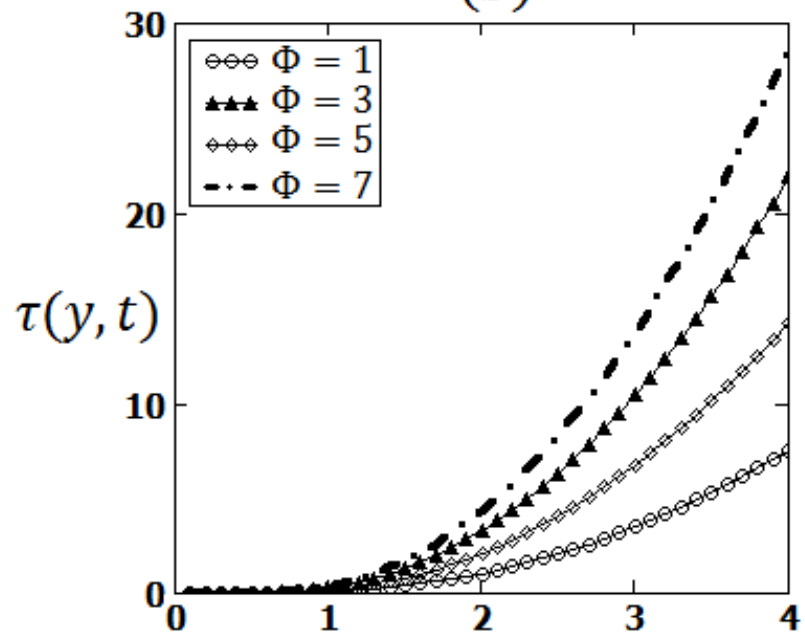

Fig. 8: Profile of velocity fields and shear stress for $\Phi$ at $U=1, t=2 S, v=1.7, \mu=11.6, \lambda_{1}=3, \lambda_{2}=3.82, \lambda_{3}=2.8, \lambda_{4}=$ 2.1, $p=1, B=5.9$ 
(a)

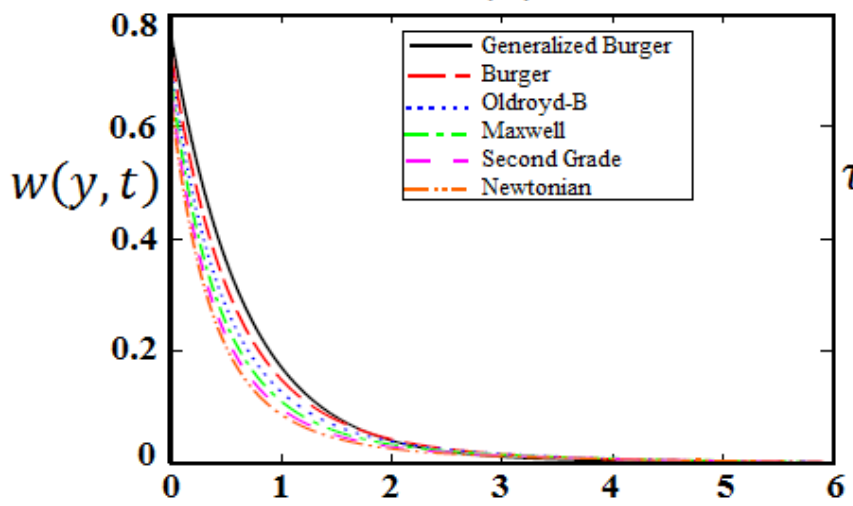

(b)

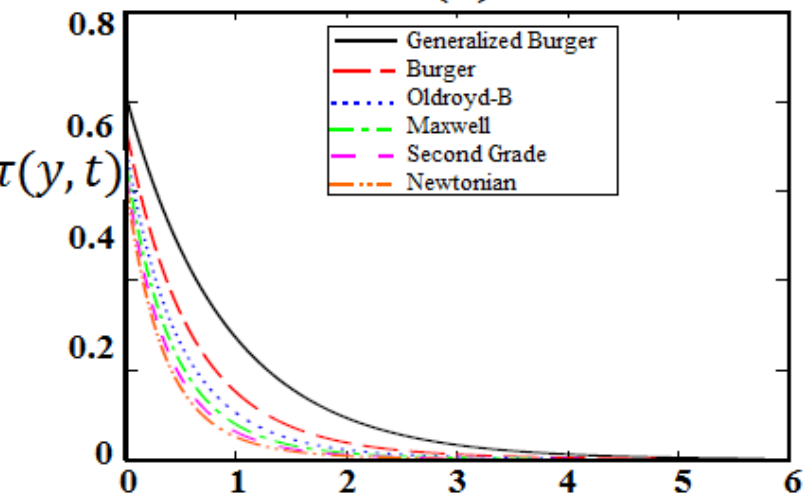

Fig. 9: Comparison of velocity fields and shear stress at $U=1, t=1 S, v=6.2, \mu=5.5, \lambda_{1}=5, \lambda_{2}=8, \lambda_{3}=4, \lambda_{4}=4.7$,

(a)

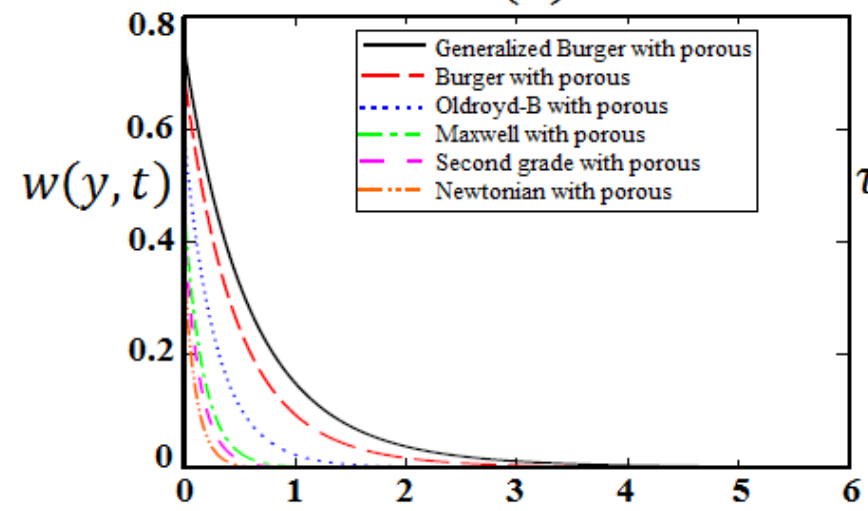

(b)

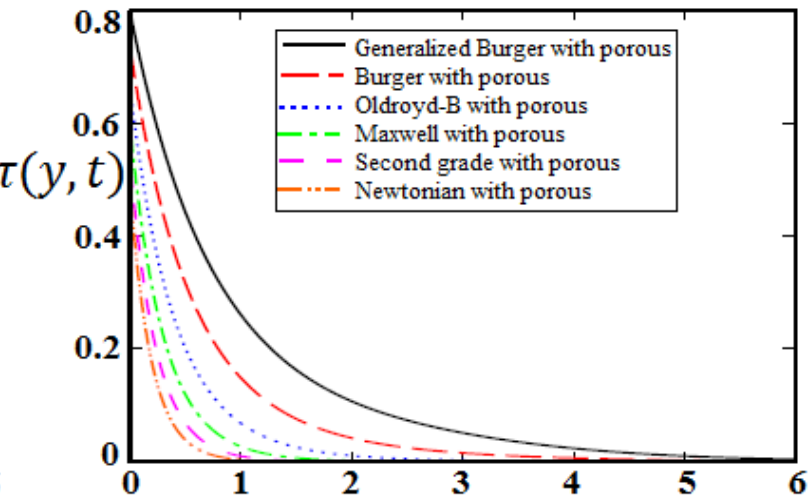

Fig. 10: Comparison of velocity fields and shear stress at $U=1, t=1 S, v=3.925, \mu=1.3, \lambda_{1}=1, \lambda_{2}=2.9, \lambda_{3}=7, \lambda_{4}=$ 27, $p=1, B=2.75, \Phi=2.5$

(a)

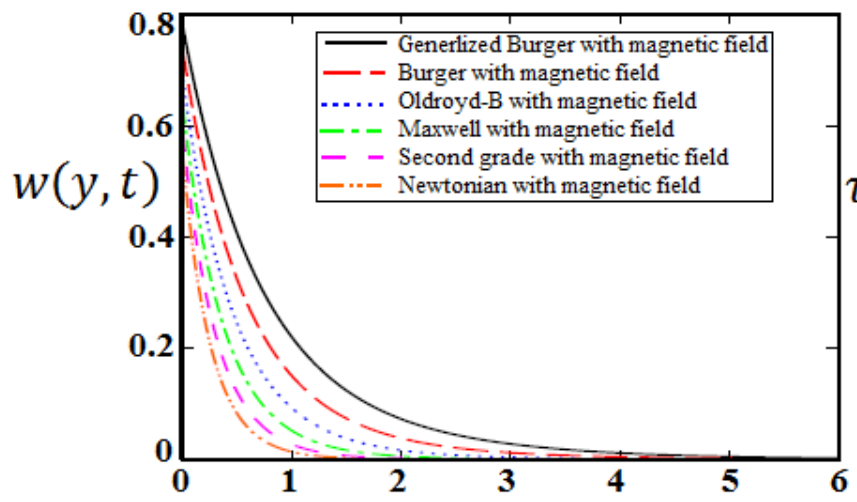

(b)

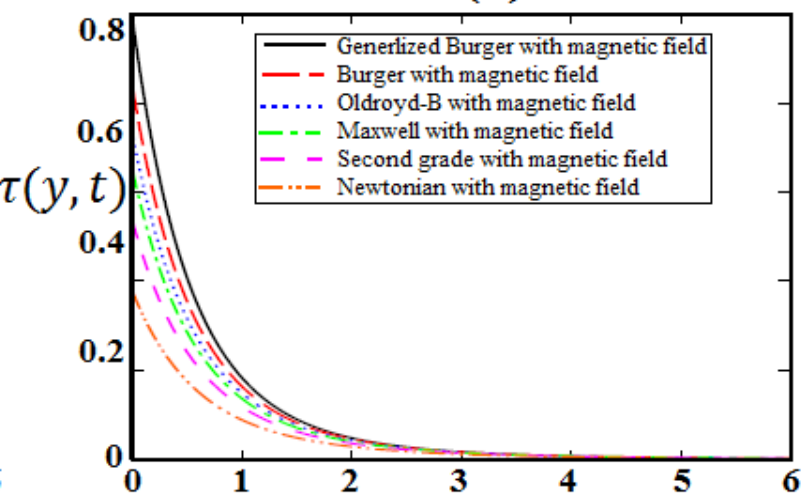

Fig. 11: Comparison of velocity fields and shear stress at $U=8, t=1 S, v=17.5, \mu=9.1, \lambda_{1}=2.5, \lambda_{2}=3, \lambda_{3}=5.7, \lambda_{4}=$ 4.2, $p=1, B=1.5, \Phi=5.25$

\section{Competing interests}

There is no conflict of interests with any person/organization upon the acceptance of this paper.

\section{Acknowledgment}

The authors Kashif Ali Abro, Mukrrum Hussain and Mirza Mahmood Baig are highly thankful and grateful to the NED University of Engineering and Technology, Karachi, Pakistan for facilitating this research work.

\section{References}

Abbasbandy S, Hayat T, Alsaedi A, and Rashidi MM (2014). Numerical and analytical solutions for Falkner-Skan flow of MHD Oldroyd-B fluid. International Journal of Numerical Methods for Heat and Fluid Flow, 24(2): 390-401.

Abro KA (2016). Porous effects on second grade fluid in oscillating plate. Journal of Applied Environmental and Biological Sciences, 6(5): 17-82.

Abro KA and Shaikh AA (2015). Exact analytical solutions for Maxwell fluid over an oscillating plane. Science International (Lahore) ISSN, 27(2): 923-929.

Ahmad K and Nazar R (2010). Magnetohydrodynamic threedimensional flow and heat transfer over a stretching surface 
in a viscoelastic fluid. Journal of Science and Technology, 3(1): 1-14.

Fetecau C, Hayat T, and Fetecau C (2008). Starting solutions for oscillating motions of Oldroyd-B fluids in cylindrical domains. Journal of non-Newtonian Fluid Mechanics, 153(2): 191-201.

Freidoonimehr N, Rashidi MM, and Mahmud S (2015). Unsteady MHD free convective flow past a permeable stretching vertical surface in a nano-fluid. International Journal of Thermal Sciences, 87: 136-145.

Hayat T, Fetecau C, and Sajid M (2008b). Analytic solution for MHD transient rotating flow of a second grade fluid in a porous space. Nonlinear Analysis: Real World Applications, 9(4): 1619-1627.

Hayat T, Khan SB, and Khan M (2008a). Exact solution for rotating flows of a generalized Burgers' fluid in a porous space. Applied Mathematical Modelling, 32(5): 749-760.

Hayat T, Najam S, Sajid M, Ayub M, and Mesloub S (2010). On exact solutions for oscillatory flows in a generalized Burgers fluid with slip condition. Zeitschrift für Naturforschung A, 65(5): 381-391.

Hsiao KL (2011). MHD mixed convection for viscoelastic fluid past a porous wedge. International Journal of Non-Linear Mechanics, 46(1): 1-8.

Hussain M, Hayat T, Asghar S, and Fetecau C (2010). Oscillatory flows of second grade fluid in a porous space. Nonlinear Analysis: Real World Applications, 11(4): 2403-2414.

Jamil M (2012). First problem of Stokes' for generalized Burgers' fluids. International Scholarly Research Network Mathematical Physics, 2012: Article ID 831063, 17 pages. https://doi.org/10.5402/2012/831063

Jamil M and Fetecau C (2010). Some exact solutions for rotating flows of a generalized Burgers' fluid in cylindrical domains. Journal of Non-Newtonian Fluid Mechanics, 165(23): 17001712.

Khan I, Ali F, and Shafie S (2012). MHD free convection flow in a porous medium with thermal diffusion and ramped wall temperature. Journal of the Physical Society of Japan, 81(4): 044401. https:// doi.org/10.1143/JPSJ.81.044401

Khan M, Hayat T, and Asghar S (2006). Exact solution for MHD flow of a generalized Oldroyd-B fluid with modified Darcy's law. International Journal of Engineering Science, 44(5): 333339.

Khan M, Iqbal K, and Azram M (2011). Closed-form solutions for MHD flow of a second-grade fluid through porous space. Special Topics and Reviews in Porous Media: An International Journal, 2(2): 125-132.

Murali Krishnan J and Rajagopal KR (2004). Thermodynamic framework for the constitutive modeling of asphalt concrete: Theory and applications. Journal of Materials in Civil Engineering, 16(2): 155-166.

Rajagopal KR (1982). A note on unsteady unidirectional flows of a non-Newtonian fluid. International Journal of Non-Linear Mechanics, 17(5-6): 369-373.

Rashidi MM, Ali M, Freidoonimehr N, Rostami B, and Hossain MA (2014). Mixed convective heat transfer for MHD viscoelastic fluid flow over a porous wedge with thermal radiation. Advances in Mechanical Engineering, 6. https://doi.org/ $10.1155 / 2014 / 735939$

Rashidi MM, and Erfani E (2012). Analytical method for solving steady MHD convective and slip flow due to a rotating disk with viscous dissipation and Ohmic heating. Engineering Computations, 29(6): 562-579.

Tan W and Masuoka T (2007). Stability analysis of a Maxwell fluid in a porous medium heated from below. Physics Letters A, 360(3): 454-460.

Tong D (2010). Starting solutions for oscillating motions of a generalized Burgers' fluid in cylindrical domains. Acta Mechanica, 214(3): 395-407.

Zhang Z, Fu C, Tan W, and Wang CY (2007). Onset of oscillatory convection in a porous cylinder saturated with a viscoelastic fluid. Physics of Fluids, 19(9): 098104. https://doi.org/ $10.1063 / 1.2773739$

Zhaosheng Y and Jianzhong L (1998). Numerical research on the coherent structure in the viscoelastic second order mixing layers. Applied Mathematics and Mechanics, 19(8): 717-723.

Zheng L, Liu Y, and Zhang X (2012). Slip effects on MHD flow of a generalized Oldroyd-B fluid with fractional derivative. Nonlinear Analysis: Real World Applications, 13(2): 513-523. 